\title{
The Digital Set-Top Box as a Virtual Channel Provider
}

\author{
Konstantinos Chorianopoulos \\ Department of Management Science and Technology \\ Athens University of Economics \& Business \\ http://itv.eltrun.aueb.gr \\ chk@aueb.gr
}

\begin{abstract}
This research is based on the realization that the desktop computing paradigm is not appropriate for television, because it is adapted to fundamentally different user aspirations and activities. Instead, the virtual channel is a model that supports the organization and dynamic presentation of digital television programming from a combination of live broadcasts, prerecorded content and Internet resources at each set-top box. The goal is to design the respective framework of user interface patterns that consider the affective nature of television usability and facilitate the diversity of viewing situations.
\end{abstract}

\section{Keywords}

Digital television, usability, affective, user interface

\section{HCI FOR THE DIGITAL SET-TOP BOX}

It is becoming apparent that an increasing proportion of home entertainment will be delivered via a set-top box in the living room. The set-top box (STB) category encompasses devices that range from MP3 jukeboxes, digital satellite receivers, and digital video recorders, to combinations of the above [8]. User interfaces for the STB make the implicit assumption that television viewers are always actively engaged. Moreover, STB UIs are designed according to the manufacturer's idiosyncrasy or by applying rules from the desktop experience.

Recent evidence has proven that traditional desktop usability principles may not transfer directly to the STB. In a usability test of video skipping interfaces, users preferred the interface that required more time and clicks, and had the highest error rate [4]. Users reasoned their choice on the basis of how fun and relaxing an interface was. Since traditional HCI settings involve a task-oriented approach where the human interacts with an application to accomplish a particular goal, computer-mediated leisure applications require a fresh view of the current paradigms.

This thesis argues that neither the vision of five hundred channels, nor the vision of a single personalized channel is suitable for giving user access to the STB. Instead, it is proposed that a small number of dynamic virtual channels may offer enough choices to cater for serendipity in media experiences, while simplifying the access to vast and diversified sources of television content. Moreover, the virtual channel model presumes only a minimal shift from the current patterns of television use [5], while it focuses this research on the design of a user interface that finetunes television programming according to the viewing situation and that considers the affective dimension of STB usability.

\section{Related Research}

The application of HCI principles to the design of the STB has been driven more by entrepreneurial spirit (TiVo) or media giant interactive TV trials (Time Warner Future Service Network) than by methodological, published, and widely available academic research [2], [3]. Past research has provided a framework for understanding the interplay between the STB technology and the family life [6], while recent research has given insights about several features, such as video skipping [4] and trust in program recommendations [9].

Strong evidence of the importance of a mental model for STB usage is provided by O'Brien et al. [6], in an ethnographic study of a digital STB trial. The concept of dynamic television program creation has been also studied before from the complementary perspective of the television program editor and producer [1]. Nevertheless, current approaches do not address the affective (relaxing and fun to use) dimension of STB usability and the diversity of viewing situations (e.g. group viewing and always-on TV coupled with spontaneous attention).

\section{VIRTUAL CHANNEL MODEL}

The ubiquity of broadcast transmissions has established a universal method for access to television programming. The television and radio channel is a familiar concept for choosing audiovisual content across all user groups. By exploiting the ubiquity and familiarity of the broadcasting mentality, the virtual channel model is proposed for access to digital STB content.

The traditional television experience has two layers: 1) the background is reserved for video play-out, while 2) the foreground is used to display overlaid information, either relevant to the background programming (e.g. music video clip information) or time critical data (e.g. CNN breaking news). However, both layers are created at the production studio and controlled at the broadcasting station. The core 
idea behind the virtual channel proposition (Figure 1) is that a part of the decision-making about television programming may shift from the media source to the STB. The television experience can now be created and controlled at the STB from a combination of local storage, real time broadcast transmissions and Internet resources, thus offering a set of virtual channels that address each STB user as an important factor in the televised content.

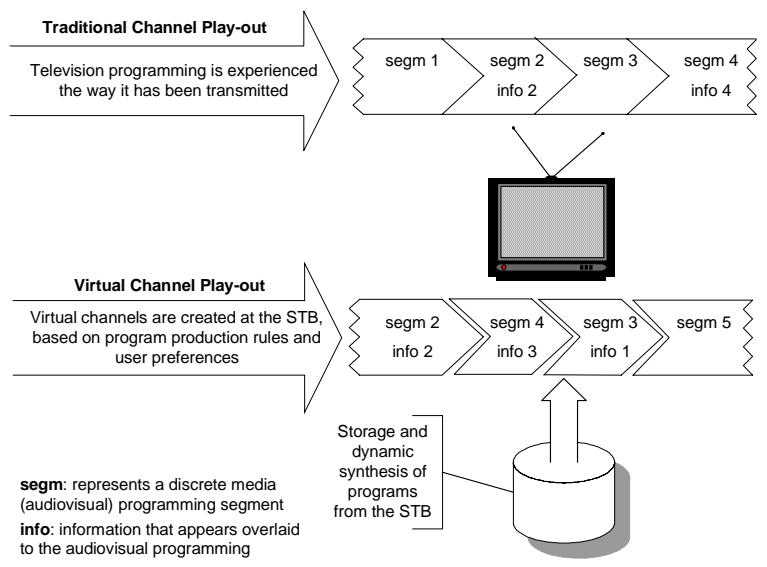

Figure 1 Generic television programming flow employing the virtual channel model, in contrast with traditional broadcasting

\section{DESIGN FOR STB APPLICATIONS}

The potential of the virtual channel model is studied through prototyping and evaluation of STB applications (advertising, music video clips, and news), employing a remote control and a TV screen in a living-room setting. The user interface design is performed on the basis of the factors that underlie the diversity of media content use in the home. These factors have been identified through an extensive literature review of the respective scientific disciplines (media consumption in home, broadcasting and consumer electronics engineering, and media content creation). The user interface evaluation, in addition to traditional usability metrics, considers the affective nature of user interaction with leisure applications.

\section{Case Studies and Significance}

The preliminary case study of television advertising although it does not feature an extensive user interfacehas demonstrated the validity of the virtual channel model. The advertising-break is created dynamically (substitute for commercials for each one of the video segments in Figure 1) from a pool of advertisement spots that have been stored on the hard disk of the STB. The exact commercials to be included on a given advertising-break are selected by the classification and targeting sub-system, which is based on media planning industry's standard procedures.

Choosing advertising as the first case study may seem controversial. On the one hand television advertising is irritating to a lot of people - research has shown that as much as $30 \%$ of people change channels during the advertising-break [7]. On the other hand, Bell and Gemmel [2] suggest dynamic advertisement insertion as a remedy to the digital rights management issues that arise when manipulating copyrighted media content in the home.

Additional case studies of important television programming include news and music video-clips. Music video-clip programming from channels such as MTV may offer a personalized 'top 20' for each week. Moreover, receiving trivial and lyrics - through Internet resourcesoffers personalization of the overlaid information. While there have been various treatments to adapt multimedia content to the user (e.g. content and collaborative filtering), the results of this research will address prominent HCI questions such as the feasibility of an agent-mediated (using MS Agent) dialog interface for leisure applications and how to evaluate a user interface when the goal is to be relaxing and fun to use.

\section{ACKNOWLEDGMENTS}

Parts of this work were supported by the IMEDIA (IST1999-11038) and CONTESSA (IST-2000-28567) projects, partially funded by the European Commission under the Information Society Technology program.

\section{REFERENCES}

1. S. Agamanolis. Isis, Cabbage, and Viper: New tools and strategies for designing responsive media. $\mathrm{PhD}$ Thesis. MIT. 2001.

2. G. Bell and J. Gemmell. A call for the home media network. CACM, 45(7):71-75, 2002.

3. J. Carey. Interactive television trials and marketplace experiences. Multimedia Tools and Applications, 5(2):207-216, 1997.

4. S. M. Drucker, A. Glatzer, S. D. Mar, and C. Wong. Smartskip: consumer level browsing and skipping of digital video content. CHI2002, pages 219-226.

5. B. Lee and R. S. Lee. How and why people watch tv: Implications for the future of interactive television. Journal of Advertising Research, 35(6), 1995.

6. J. O'Brien, T. Rodden, M. Rouncefield, and J. Hughes. At home with the technology: an ethnographic study of a set-top-box trial. ACM TOCHI, 6(3):282- 308, 1999.

7. L. van Meurs. Zapp! a study of switching behavior during commercial breaks. Journal of Advertising Research, 38(1), 1998.

8. P. Wallich. Digital hubbub. IEEE Spectrum, pages 2631, July 2002.

9. J. Zimmerman and K. Kurapati. Exposing profiles to build trust in a recommender. CHI2002 Extended Abstracts, pages 608-609, 2002. 